\title{
The intellectual disability protein Oligophrenin1 controls astrocyte morphology and migration
}

Running title: Structural and migratory alterations in astrocytes deficient for OPHN1 via the ROCK pathway

Laure-Elise Pillet*1,2,3, Noémie Cresto*1, Yoann Saillour ${ }^{3,6}$, Grégory Ghézali ${ }^{1}$, A. Bemelmans $^{4}$, Jean Livet ${ }^{5}$, Thierry Bienvenu ${ }^{3,6}$, Nathalie Rouach ${ }^{1 \$}$, Pierre Billuart ${ }^{3,6 \$}$

${ }^{1}$ Center for Interdisciplinary Research in Biology, Collège de France, CNRS UMR 7241, INSERM U1050, Labex Memolife, PSL Research University, Paris 75005, France.

${ }^{2}$ Doctoral School N ${ }^{\circ} 562$, Paris Descartes University, Paris 75006, France

${ }^{3}$ Institut Cochin, INSERM UMR 1016, CNRS UMR 8104, Université de Paris, F-75014, Paris, France.

${ }^{4}$ Commissariat à l'Energie Atomique et aux Energies Alternatives (CEA), Département de la Recherche Fondamentale, Institut de biologie François Jacob, MIRCen, and CNRS UMR 9199, Université Paris-Sud, Neurodegenerative Diseases Laboratory, Fontenay-aux-Roses 92260, France.

${ }^{5}$ Sorbonne Université, INSERM, CNRS, Institut de la Vision, 75012 Paris, France.

${ }^{6}$ Present address: Institut de Psychiatrie et de Neurosciences de Paris (IPNP), UMR 1266, INSERM, Université de Paris, 102-108 rue de la Santé, F-75014, Paris, France.

${ }^{*}$ These authors contributed equally to this work

$\$$ These authors contributed equally to this work

`Corresponding authors: Pierre Billuart, pierre.billuart@inserm.fr; Nathalie Rouach, nathalie.rouach@college-de-france.fr

\section{Conflict of Interest Statement}

The authors declare no competing financial interests.

\section{Data availability Statement}

The data that support the findings of this study are available from the corresponding author upon reasonable request.

\section{Acknowledgments}

We thank Chloé Delépine, who provided us with lysats from hiPSC differentiated in astrocytes in vitro, and for her kind help. We also thank Pascal Ezan for his help.

Research was supported by the Jerome-Lejeune Foundation (2015b/FJL1415, https://www.fondationlejeune.org) to P.B., the French National Agency for Research (ANR 15-CE16-0019-01) to P.B. and N.R., the European Research Council (Consolidator grant \#683154) and European Union's Horizon 2020 research and innovation program (Marie Sklodowska-Curie Innovative Training Networks, grant \#722053, EU-GliaPhD) to N. R., and the BioSPC doctoral school to L.-E.P.

\section{Word Count}

Total: 8348

Abstract: 144

Introduction: 746 
Material and Methods: 1684

Results: 1531

Discussion: 1098

References: 2018

Figure legends: 1127

\begin{abstract}
Astrocytes are involved in several aspects of neuronal development and properties which are altered in intellectual disability. Oligophrenin1 is a RhoGAP protein implicated in actin cytoskeleton regulation, and whose mutations are associated with X-linked intellectual disability. Oligophrenin1 is expressed in neurons, where its functions have been widely reported at the synapse, as well as in glial cells. However, its roles in astrocytes are still largely unexplored. Using in vitro and in vivo models of oligophrenin1 disruption in astrocytes, we found that oligophrenin1 regulates at the molecular level the RhoA/ROCK/MLC2 pathway in astroglial cells. We also showed at the cellular level that oligophrenin 1 modulates astrocyte morphology and migration both in vitro and in vivo, and is involved in glial scar formation. Altogether, these data suggest that oligophrenin 1 deficiency alters not only neuronal but also astrocytic functions, which might contribute to the development of intellectual disability.
\end{abstract}

\title{
Keywords
}

Intellectual disability, Oligophrenin-1, RhoA GTPase, astrocyte, morphology, migration

\section{Main points:}

- Loss of function of the OPHN1 intellectual disability protein increases RhoA GTPase signaling in astrocytes

- OPHN1 controls glial scar formation in vitro and in vivo

\section{MAIN TEXT}

\section{Introduction}

Intellectual disability (ID) is a neurodevelopmental disorder establishing before the age of 18 and describing problems in intellectual functioning (IQ below 70) and adaptative behavior including social, conceptual and practical abilities (Milani, Ronzoni, \& Esposito, 2015). The pathology concerns 1 to $2 \%$ of the worldwide population, is often misdiagnosed and involves very high costs for the healthcare system (Salvador-Carulla et al., 2011), pointing to the need to develop new therapeutical approaches for this neurological disease.

The causes of ID are complex and include environmental and genetic origins. The second most frequent form of genetic ID in males, after Down syndrome, results from mutations in a chromosome X-linked gene (Ropers \& Hamel, 2005). A large fraction of ID genes are coding for proteins involved in synapse function and it appears that the common feature of ID, and more specifically X-linked ID (XLID), is a dysfunction of the synapse (Humeau, Gambino, Chelly, \& Vitale, 2009; Pavlowsky, Chelly, \& Billuart, 2012; Valnegri, Sala, \& Passafaro, 2012). 
Several identified genes implicated in XLID are involved in the regulation of the RhoGTPases (Ba, van der Raadt, \& Nadif Kasri, 2013; Zamboni et al., 2018), which are small GTPase proteins of the Rho family that play a critical role in cytoskeleton remodeling. They regulate different cellular processes such as migration, adhesion and morphology (EtienneManneville \& Hall, 2002; Raftopoulou \& Hall, 2004; Ridley, 2001). RhoGTPases play an important role in neuronal survival, axon formation and dendritic maturation (Auer, Hausott, \& Klimaschewski, 2011; Govek, Newey, \& Van Aelst, 2005; Stankiewicz \& Linseman, 2014) and their implication in synaptopathy is today largely explored (Nadif Kasri \& Van Aelst, 2008; Newey, Velamoor, Govek, \& Van Aelst, 2005; San Martin \& Pagani, 2014). Oligophrenin gene, which encodes the oligophrenin1 (OPHN1) RhoGAP protein was first identified in a female patient showing mild ID with a balanced $\mathrm{X}$ autosome translocation (Billuart et al., 1998). Different case reports were published through the years describing familial OPHN1 mutations leading to a specific syndromic XLID with cerebellar hypoplasia and ventriculomegaly (Moortgat et al., 2018). OPHN1 knockout (KO) mice are today considered as a mouse model for this pathology as they present a human-like syndromic form of XLID. These mice indeed display an enlargement of ventricles, defects in working and spatial memory as well as social interactions and hyperactivity (Khelfaoui et al., 2007). OPHN1 is a negative regulator of RhoGTPases and amongst them stimulates the GTPase activity of RhoA to its GDP bound inactivated form and turns-off the signaling from RhoA to RhoA-associated protein kinase (ROCK) and myosin light chain 2 (MLC2) (Fig. 1b). OPHN1 protein binds to F-actin and is expressed at high level in neurons, but also in glial cells during development and adulthood (Fauchereau et al., 2003). Its role in different steps of neuronal network maturation is today well established. OPHN1 regulates neurogenesis, dendritic spine maturation, axon guidance and synaptic plasticity (Allegra et al., 2017; Compagnucci et al., 2016; Nadif Kasri, Nakano-Kobayashi, Malinow, Li, \& Van Aelst, 2009; Nakano-Kobayashi, Tai, Nadif Kasri, \& Van Aelst, 2014; Redolfi et al., 2016).

In vitro and in vivo pharmacological treatment targeting the RhoGTPase signaling pathway partly rescues the morphological defects of neurons as well as the behavioural alterations in OPHN1 models of ID, implying that multiple therapeutic approaches may be combined to improve ID conditions (Allegra et al., 2017; Meziane et al., 2016). The emerging view on the development of neurological diseases is that they involve other brain cells than neurons, and in particular astroglia (Chung, Welsh, Barres, \& Stevens, 2015; Dallerac \& Rouach, 2016; Dossi, Vasile, \& Rouach, 2018). Astrocytes are indeed implicated in neuronal maturation, pruning, synaptic activity and plasticity (Bosworth \& Allen, 2017; Vasile, Dossi, \& Rouach, 2017). Moreover, their contribution to different synaptic pathologies such as autism or Down syndrome was already pointed out (Araujo et al., 2018; Cresto, Pillet, Billuart, \& Rouach, 2019; Petrelli \& Bezzi, 2018). Studying the role of OPHN1 in astrocytes could thus permit to better describe the astrocytic contribution to XLID and to understand the other underlying cellular mechanisms of this pathology.

For this purpose, we here used Ophn1 deficient mice to study astrocytic morphology and migration in vitro and in vivo. We report that OPHN1 is expressed in human and murine cultured astrocytes and that ROCK activity, dependent of RhoGTPases activation, is higher in astrocytes deficient for OPHN1 in vitro. Moreover, cellular processes regulated by RhoGTPases such as migration and morphology are also dysregulated at the cellular and physiological levels in these astrocytes. 


\section{Materials and Methods}

\section{OPHN1 knockout mice}

We used male Ophn1 $1^{-/ y}$ (KO: B6.129P-Ophn1 $1^{\text {tm1Bill }}$ ) and Ophn ${ }^{\text {lox/y }}$ mice (loxKO: B6.129POphn $1^{\text {tm2Bill }}$ ) (Khelfaoui et al., 2007) for all experiments and their control littermates Ophn $1^{+/ y}$ or wild type (WT) mice with the same genetic background. C57BL/6NRj were ordered from Janvier Labs. Tg(GFAP-Cre/ERT2)1Fki mouse line were crossed to Ophn1 loxKO in order to generate the conditional KO model used in the cell culture experiments (Hirrlinger, Scheller, Braun, Hirrlinger, \& Kirchhoff, 2006).

All applicable international, national, and/or institutional guidelines for the care and use of animals were followed. Adult mice were housed under a $12 \mathrm{~h}$ light $/ 12 \mathrm{~h}$ dark circle, with ad libitum access to food and water, in accordance with European Community (Directive 201063/ EEC) and French regulations (Code Rural R214/87-130). Experimental procedures were approved by the local ethics committee and registered with the French Research Ministry (CEEA, Paris 6).

\section{Primary culture of astrocytes}

Primary astrocyte cultures of cerebral cortex were prepared from 1 to 3-day-old mice according to previously described methods (Ghezali et al., 2018). Briefly, after dissection and removal of the meninges, the whole cortexes were gently mechanically dissociated with a P1000 pipette in cold PBS supplemented with 1\% glucose. Cells were then collected by centrifugation at $800 \mathrm{~g}$ for $5 \mathrm{~min}$, resuspended in Dulbecco's Modified Eagle Medium (DMEM, 21885025, Thermofisher) supplemented with 10\% heat-inactivated Fetal Bovine Serum (FBS) and penicillin-streptomycin (1\% final), plated in Petri dishes coated with polyornithine (P4957, Sigma-Aldrich) for molecular biology experiments or glass coverslips for microscopy and immunostaining. Incubation was at $37^{\circ} \mathrm{C}$ in a humidified atmosphere of 95\% air, 5\% $\mathrm{CO}_{2}$. After reaching confluence, astrocytes were treated with cytosine arabinofuranoside (AraC) for $24 \mathrm{~h}$ to avoid the proliferation of microglial cells. Medium was changed 2 times per week. Around DIV25, confluent astrocytes were starved overnight with DMEM medium without FBS for in vitro experiments to reduce RhoA activation. Cre-ERT2 expressing loxKO astrocytes were treated with 4-hydroxy-Tamoxifen (4-OHT at $0.1 \mu \mathrm{M})$ during 1 or 4 consecutive days to induce a reduction in OPHN1 expression.

\section{In vitro scratch-induced migration assay}

Confluent astrocytes were wounded by scraping monolayers with a $10 \mu$ pipette tip at DIV 25 after overnight serum starvation. To quantify scratch-induced migration of astrocytes, the distance traveled by the cells in 24 hours was measured using phase contrast pictures taken at the beginning and 24 hours after the scratch. To ensure that the pictures taken $24 \mathrm{~h}$ after the wound were taken at the same place, two perpendicular wounds were performed, and four pictures were taken at the crossing area. To quantify migration, we measured an index based on the difference between areas measured $0 \mathrm{~h}$ and $24 \mathrm{~h}$ after migration normalized to the wound length. This index was normalized with mean WT migration index in unscratched conditions. Then after $24 \mathrm{~h}$, cells were washed with PBS $1 \mathrm{X}$ and fixed $15 \mathrm{~min}$ at room temperature with $4 \%$ paraformaldehyde for immunostaining. Glass coverslips were then stored in PBS at $4^{\circ} \mathrm{C}$. Images were taken using a phase contrast microscope at a10X magnification.

For biochemical analysis, several wounds were made in multiple directions ( 15 vertically and 15 horizontally) on confluent astrocytes. $20 \mathrm{~min}$ or $24 \mathrm{~h}$ later, cells were lysed in $1 \mathrm{X}$ sample buffer containing $5 \%$ of B-Mercaptoethanol, $0.45 \mathrm{M}$ dithiothreitol (DTT), $1 \%$ sodium dodecyl sulfate (SDS), $0.4 \mathrm{M}$ Tris, $50 \%$ glycerol, $0.2 \%$ bromophenol blue. Samples were then 
denaturized $10 \mathrm{~min}$ at $100^{\circ} \mathrm{C}$, sonicated during $5 \mathrm{~min}$, aliquoted to avoid thawing and freezing cycles and stored at $-80^{\circ} \mathrm{C}$.

\section{Treatment with a ROCK inhibitor Y-27632}

For rescue experiments, cells were incubated with $0.1 \mu \mathrm{M}$ of the ROCK inhibitor Y27632 (Y0503, Sigma-Aldrich). Around DIV25, confluent astrocytes were starved overnight with DMEM medium without FBS. The next day, half of the medium was removed and replaced by a medium without FBS containing dimethylsulfoxyde (DMSO) or the inhibitor of ROCK, Y-27632 $(0.1 \mu \mathrm{M}$ final $)$, diluted in DMSO. Immediately after the treatment, astrocytes were wounded by scraping monolayers with a $10 \mu \mathrm{l}$ pipette tip. Cells were lysed or fixed $24 \mathrm{~h}$ after the treatment for biochemistry and microscopy analysis.

\section{Western Blot}

10 to $20 \mu 1$ of total protein extract in sample buffer $1 \mathrm{X}$ were loaded on a 8-12\% SDS-PAGE gel (NuPAGE Novex Bis-Tris gel, Invitrogen) and transferred on nitrocellulose membrane (Transfer kit from Invitrogen) before hybridization with various primary antibodies (See below). Data were normalized with WT mean in basal conditions and statistical comparisons between WT and KO astrocytes and between different conditions were performed. Human proteins (hAstro) were extracted from cultured astrocytes after the differentiation of induced pluripotent stem cells (iPSC) from a Rett patient isogenic cell clone expressing the WT methyl CpG binding protein 2 (MeCP2) allele (Delepine et al., 2016; Williams et al., 2014).

\section{Antibodies}

We used the antibodies against: MLC2 (Cell Signaling, Rabbit, reference 8505S) at 1:500, PMLC2 (Ser19) (Cell Signaling, Rabbit, reference 3671S) at 1:500, Nestin (Merck-Millipore, Mouse, reference MAB353) at 1:200, GFAP (Sigma-Aldrich, Mouse, reference G3893) at 1:500, Vinculin (Invitrogen, Rabbit, reference 42H89L44) at 1:200, Phospho-histone H3 (Ser10) (Millipore, Rabbit, reference 06-570) at 1:200, cleaved Caspase3 (Cell Signaling, Rabbit, reference 9661) at 1:200, GFP (green fluorescent protein, Abcam, Chicken, reference ab13970) at 1:500, GLT-1 (glutamate-transporter-1, Frontier Institute, Rabbit, reference Af670) at 1:500, GAPDH (Glyceraldehyde-3-phosphate dehydrogenase, Sigma-Aldrich, reference G9295 at 1:10 000), OPHN1 (crude Rabbit serum (Fauchereau et al., 2003)) at 1:500, Glutamine synthase (BD transduction laboratories, Mouse, reference 45020) at 1:500, S100ß (Sigma-Aldrich, Rabbit, reference S2644) AT 1:500.

\section{Adeno-associated virus production}

A transgene composed of GFP in a single open reading frame was placed under the control of a glial fibrillary acidic protein (GFAP)-specific promoter in an adeno-associated virus (AAV) shuttle plasmid containing the inverted terminal repeats (ITR) of AAV2 (Ghezali et al., 2018) (Fig. 5a). Pseudotyped serotype 9 AAV particles were produced by transient co-transfection of HEK-293T cells, as previously described (Berger et al., 2015). Viral titers were determined by quantitative PCR amplification of the ITR on DNase-resistant particles and expressed as vector genomes vector genome per $\mathrm{ml}(\mathrm{vg} / \mathrm{ml})$.

\section{AAV injection and in vivo glial scar formation}

Adult mice weighting $\sim 20 \mathrm{~g}$ were deeply anaesthetized by a mixture of ketamine $(75 \mathrm{mg} / \mathrm{kg})$ and xylazine $(5 \mathrm{mg} / \mathrm{kg})$ and placed in a stereotaxic frame. Injury was bilaterally made in the cortex with a 34-gauge blunt-tipped needle linked to a $2 \mu 1$ Hamilton syringe by a polyethylene catheter at the following stereotaxic coordinates: AP:-0.9mm; L: $-2 \mathrm{~mm}$ to the bregma at a depth of $-2.75 \mathrm{~mm}$ to the skull. The needle was left in place for five minutes and 
was slowly withdrawn. To observe the morphology of astrocytes in the wounded area, the needle used to perform the injury was also used to deliver AAV expressing a cytoplasmic GFP protein under the GFAP promoter (see above) at a rate of $0.25 \mu 1 / \mathrm{min}$ with an automatic pump, $1 \mu 1$ of AAV at $1 \times 10^{10}$ viral particles into each site. Animals were sacrificed 15 days after injury.

\section{In utero electroporation}

To label astrocytes fine processes and visualize their territorial domains, we synthetized a ${ }^{P B} G f a:: L c k-G F P$ transposable transgene expressing a membrane-tethered form of GFP (LckEGFP) from the Gfap ABC(1)D promoter (Lee, Messing, Su, \& Brenner, 2008). As in (Colin et al., 2009), the 3'UTR of the transgene also included a woodchuck hepatitis virus posttranscriptional regulatory element (WPRE) to enhance mRNA processing, and four tandem miR124 target sequences to inhibit neuronal expression, placed upstream of the transcription terminator (bGH pA) (Colin et al., 2009). To enable genomic integration in astrocyte progenitors, the cassette was framed by $5^{\prime}$ and $3^{\prime}$ minimal terminal repeats of the piggyBac transposon (Meir et al., 2011) with an additional $3 \mathrm{bp}$ from the wild type transposon in the 3' TR as in (Loulier et al., 2014). Map of the ${ }^{P B} G f a:: L c k-G F P$ transgene is shown in Fig 4a. Full sequence is available upon request. This vector was co-electroporated $(1 \mu \mathrm{g} / \mu \mathrm{L}$ of each plasmid) in E15.5 Swiss; C57BL/6NRj mixed background mouse embryos together with a CAG-driven plasmid expressing a hyperactive form of piggyBac transposase (Yusa, Zhou, Li, Bradley, \& Craig, 2011), an approach yielding semi-sparse labeling of protoplasmic astrocytes (Chen \& LoTurco, 2012). Anesthetized mice at postnatal day 21 were perfused with $4 \%$ paraformaldehyde (PFA) in $0.1 \mathrm{M}$ Phosphate buffer and dissected brain were processed for vibratome sectioning $(30 \mu \mathrm{m})$ and immunostaining for GFP, GS and S100ß markers. Images were taken using an inverted confocal microscope (Leica SP5) at a 40X magnification.

\section{Immunohistochemistry}

Mice were deeply anesthetized with Thiopental (Pentothal) and perfused intracardially with PFA $2 \%$. The brains were carefully dissected and post-fixed overnight with PFA $2 \%$ at $4{ }^{\circ} \mathrm{C}$ and were sliced with a freezing microtome. The next day, PFA was removed and the brains were cryoprotected overnight in phosphate buffer saline (PBS) containing 30\% of sucrose. The brains were the cut into $30 \mu \mathrm{m}$ sections on a freezing microtome (Leica, Germany). Slices were stored in PBS at $4^{\circ} \mathrm{C}$ until use. For immunohistochemistry, slices were blocked with PBS-Gelatin-Triton (PBS with $0.002 \%$ of gelatin and $0.25 \%$ of Triton), for $1 \mathrm{~h}$ at room temperature, incubated overnight at $4{ }^{\circ} \mathrm{C}$ with primary antibodies in the blocking solution, washed three times with PBS, incubated $1 \mathrm{~h} 30$ with secondary antibodies with DAPI in the blocking solution and washed three times with PBS before mounting with Fluoromount (Invitrogen). In our in vivo model of wound, DAPI, Caspase 3 and Phosphohistone3 positive nucleus were counted within an area around the wound that was the same for all pictures. DAPI positive nucleus density was normalized with WT mean. The number of Caspase 3 and Phosphohistone3 positive cells was normalized to surface area. This ratio was normalized to WT mean. Nestin area was measured by applying the same threshold to all images and normalized to WT mean.

\section{Sholl analysis}

Z-stack images were acquired at a confocal microscope with a 40X magnification (Leica SP5 inverted confocal). Isolated astrocytes were selected based on their GFP staining. The ImageJ plugin 'Sholl analysis' (Abramoff, Megalhães, \& Ram, 2004) was used to measure the 
number of intersections between GFP staining and the concentric circles spaced by $5 \mu \mathrm{m}$ and centered on astrocyte nucleus.

\section{Statistical analysis}

All data are expressed as mean \pm s.e.m. Statistical significance for within-group comparisons was determined by one- or two-way ANOVA, whereas unpaired t-tests or Mann-Whitney tests were used for between-group comparisons, unless otherwise stated. Frequency distributions were compared using Kolmogorov-Smirnov test (KS test). Statistical analysis was performed using Prism (Graphpad software, CA USA).

\section{Results}

\section{OPHN1 regulates in vitro Rho-associated protein kinase activity in migrating astrocytes}

We first investigated whether OPHN1 is expressed in vitro in human and murine astrocytes. We found by Western Blot that human astrocytes differentiated from induced pluripotent stem cells (iPSC) express OPHN1 similarly to rodent astrocytes (Fig. 1a) (Delepine et al., 2016; Fauchereau et al., 2003). Western blot analysis also showed that OPHN1 was however absent in primary cultures of astrocytes from OPHN1 KO mice (Fig. 1a), indicating that this in vitro model of OPHN1 disruption is relevant to study the role of OPHN1 in astrocytes.

Since we and others previously reported that OPHN1 negatively regulated the GTPase RhoA in various cell types to control ROCK activity, one of the most studied effector of RhoA, we investigated ROCK activation in astrocytes during migration (Govek et al., 2004; Khelfaoui et al., 2009). To estimate its activation, we quantified by Western Blot the phosphorylation level of MLC2, one of its direct targets, involved in actin-myosin contraction and migration process (Fig. 1b). First, we confirmed that ROCK activity is increased in unscratched conditions (Khelfaoui et al., 2009). Similarly to the myosin phosphatase target subunit 1 (MYPT1) (Khelfaoui et al., 2009), another target of RhoA/ROCK pathway implicated in cell migration (Matsumura \& Hartshorne, 2008), we found that the phosphorylation level of MLC2 in confluent astrocytes was increased by $\sim 30 \%$ in astrocytes lacking OPHN1 (WT: $n=5$; KO: $\mathrm{n}=6, \mathrm{p}=0.0303$, Mann-Whitney-test, Fig. 1c,d), whereas MLC2 content was not different (WT: $n=5$; KO: $n=3, p=0.98$, Mann-Whitney-test, Fig. S1). We then tested whether MLC2 phosphorylation level varies during migration in our in vitro model of migrating astrocytes (Fig. 1c). We found that MLC2 phosphorylation levels do increase over time in migrating astrocytes from WT mice but not from OPHN1 KO mice (WT: $n=5 ;$ KO: $n=6 ; p=0.0174$, twoway ANOVA, $\mathrm{t}=0$ vs $\mathrm{t}=24 \mathrm{~h}$ Bonferroni's post-hoc test $\mathrm{p}=0.0056$, Fig. 1e). Next we used another in vitro model, in which we acutely turned-off the expression of OPHN1 using the conditional mouse allele of Ophn1 gene (loxKO) (Khelfaoui et al., 2007). Upon hydroxytamoxifen (4-OHT) treatment of Cre-ERT2 expressing loxKO astrocytes during one or four days, we observed a reduction in OPHN1 expression (WT: $n=3 ; K O: n=3, p=0.0264$, ANOVA-test, and $\mathrm{p}=0.0283$ for Bonferroni's post-hoc test comparing untreated vs 4 days, green bars) and a parallel increase in MLC2 phosphorylation (WT: $n=3 ; K O: n=3, p=0.0001$, ANOVA-test, and $p=0.0006$ for Bonferroni's post-hoc test comparing untreated vs 4 days and $\mathrm{p}=0.0001$ for Bonferroni's post-hoc test comparing 1 day vs 4 days of 4-OHT treatment, blue bars) (Fig. 1f, g), indicating that the RhoA/ROCK activation is a direct consequence of OPHN1 loss-of-function.

Since OPHN1 disruption over-activates RhoA in unstimulated conditions, these results, taken together, suggest that OPHN1 constitutively inactivates RhoA in astrocytes. This leads to an alteration of ROCK phosphorylation pathways, which should be activated in migrating astrocytes. 


\section{OPHN1 regulates astroglial migration}

Since RhoGTPases are involved in cell migration (Ridley, 2015; Zegers \& Friedl, 2014), we investigated whether OPHN1 regulates migration of astrocytes. To evaluate migration in vitro, we performed the wound healing assay on confluent astrocytes (Etienne-Manneville, 2006). We found that OPHN1 KO astrocytes migrated less than WT astrocytes $24 \mathrm{~h}$ after the wound, as the scar area was larger in astrocyte cultures lacking OPHN1 in comparison to control conditions (WT: $n=9 ; \mathrm{KO}: \mathrm{n}=11 ; \mathrm{p}=0.0041$ for ANOVA-test, and $\mathrm{p}=0.0210$ for Bonferroni's post-hoc test comparing WT(-) to $\mathrm{KO}(-)$ conditions, Fig.2 a-b). Since we found a higher ROCK activity in astrocytes from OPHN1 KO mice, we then tested whether the alteration in migration could be rescued by inhibiting ROCK activity in OPHN1 KO astrocytes. We found that partial inhibition of ROCK $(\mathrm{Y} 27632,0.1 \mu \mathrm{M})$ rescued normal migration in OPHN1 KO astrocytes $(n=11, p=0.0430$ for Bonferroni's post-hoc test comparing $\mathrm{KO}(+)$ to $\mathrm{KO}(-)$ conditions and $\mathrm{p}=0.8143$ for Bonferroni's post-hoc test comparing $\mathrm{WT}(+)$ to $\mathrm{KO}(+)$ conditions, Fig. $2 b)$, while it had no effect in WT astrocytes $(n=9, p>0.9999$, Bonferroni's post-hoc test comparing WT(+) to WT(-) conditions, Fig. 2b). In addition, we found that Y27632 significantly reduced the MLC2 phosphorylation in KO ( $n=6$, ANOVAtest, $\mathrm{p}=0.0136$, and $\mathrm{p}=0.0118$ for Bonferroni's post-hoc test comparing $\mathrm{KO}(+)$ to $\mathrm{KO}(-)$ conditions) with no effect on WT conditions ( $p=0.0007$ for ANOVA-test and $p>0.9999$ for Bonferroni's post-hoc test, comparing WT(+) to WT(-) conditions (see Fig. S2)) (Fig. 2c,d). These data suggest that over-activation of the ROCK pathway is involved in the migration defect of OPHN1 KO astrocytes.

\section{OPHN1 regulates in vitro the morphology of migrating astrocytes}

The first stage of cell migration results in an elongation of the cell before actual migration, corresponding to the nucleus movement through the retraction of the filipodia. Since OPHN1 regulates the actin cytoskeleton (Fauchereau et al., 2003), we investigated whether this elongation was altered in astrocytes lacking OPHN1. We labeled migrating astrocytes with vinculin, a protein at the interface of membrane and cytoskeleton, which delineates their contours (Fig. 3a). We found that OPHN1 KO astrocytes at the migration front had a smaller cell area than WT astrocytes (WT: $n=320 ; K O: n=402, p=0.0027$, t-test with Welch's correction; $p=0.0032$, KS test). This effect was specific to migrating astrocytes at the front, since confluent WT and OPHN1 KO astrocytes at the rear showed similar morphology (Fig. S3). OPHN1 KO astrocytes also showed a smaller major axis (WT: $n=320 ; \mathrm{KO}$ : $\mathrm{n}=402$; $p=0.0002$, t-test with Welch's correction; $p=0.012$, KS test) with no difference in minor axis length in comparison to WT astrocytes (WT: $n=320 ; \mathrm{KO}: \mathrm{n}=402 ; \mathrm{p}=0.4358$, t-test with Welch's correction; $p=0.7009$, KS test, Fig. $3 b-c)$. This observation implies that the difference in cell area between WT and OPHN1 KO astrocytes results from an altered elongation of astrocytes at the leading edge.

\section{OPHN1 contributes to astroglial scar formation}

We then investigated in vivo the effect of OPHN1 loss-of function on astroglial morphology in physiological conditions. Progenitors of astrocytes were electroporated at embryonic stage E15.5 with a novel integrative vector (Fig. 4a) that allows the specific expression of membrane tagged GFP in Glutamine Synthase (GS) and S100ß positive astrocytes (Fig. 4b). Since we previously studied in vitro astrocytes differentiated from cortical progenitors, we here investigated the morphological complexity of GFP-positive astrocytes from the somatosensory cortex at P21 by performing Sholl analysis to estimate their ramification. Astrocytes from OPHN1 KO and WT astrocytes show similar complexity (WT: $n=23$; KO: $\mathrm{n}=29, \mathrm{p}=0.5846$ for two-way ANOVA, Fig. $4 \mathrm{c}$ ) 
Then, we took advantage of a model of glial scar formation obtained after mechanical injury in the cortex to study migration in vivo (Sofroniew, 2009; Suzuki, Sakata, Kato, Connor, \& Morita, 2012). Focal injury was induced by a needle placed stereotaxically in the somatosensory cortex (Fig. 5a). To investigate the whole-cell morphology of astrocytes near the scar region, we specifically labeled astrocytes by taking advantage of the needle to simultaneously micro-inject an AAV expressing GFP under the GFAP promoter (Ghezali et al., 2018). We observed isolated astrocytes expressing the cytoplasmic GFP protein (Fig. 5b). Using Sholl analysis on GFP-positive astrocytes (Fig. 5c), we found that KO astrocytes were less ramified than WT astrocytes, with a more severe phenotype in the proximal branches around $15 \mu \mathrm{m}$ from the nucleus (WT: $\mathrm{n}=30$; KO: $\mathrm{n}=30$, ANOVA genotype $\mathrm{p}<0.0001$, Bonferroni's post-hoc test $\mathrm{p}=0.0169 \mathrm{KO}$ versus WT at $15 \mu \mathrm{m}$ ).

As previously described (Suzuki et al., 2012), we defined two specific parts of the astrocytic scar: the proximal region of the wound, where astrocytes are strongly polarized and orientate their processes toward the wounded tissue, and the distal region, where astrocytes are round and extend their processes in all directions. To delimitate the proximal region where fibers of projected astrocytic processes are exclusively labeled, we performed immunohistochemistry for nestin, an intermediate filament protein whose expression is re-induced in the adult during glial scar formation. We focused our observations in the wounded area where astrocytic fibers expressing nestin are present all around the injured tissue (Fig. 6a). We found that the proximal region of the scar defined by nestin expression (Fig. 6b) was smaller in OPHN1 KO mice than in WT mice (WT: $n=5$; KO: $n=5, p=0.0335$, t-test, Fig. 6b). Altogether, these data show that the glial scar formation is altered in OPHN1 deficient mice.

We then investigated whether the alteration in glial scar formation resulted from a change in cell density, apoptosis or proliferation. To do so, we performed immunohistochemistry using specific markers of cell nucleus (DAPI), cell apoptosis (caspase 3, a perinuclear protein) or cell proliferation (phopho-histone3, a nuclear protein) (Fig. 6c-d). Cell density was not different in WT and OPHN1 KO mice (WT: $n=5 ; \mathrm{KO}: \mathrm{n}=4, \mathrm{p}=0.2892$ for t-test, Fig. $6 \mathrm{c}$ ). The number of caspase 3 positive nuclei or phosphohistone 3 positive nuclei were also not different in the wounded area of WT and KO mice (WT: $n=5 ; K O: n=4$, caspase $3: p=0.6250$, t-test; phospho-histone3: $\mathrm{p}=0.5090$, t-test, Fig. $6 \mathrm{c}-\mathrm{d}$ ), indicating no difference in apoptosis and proliferation between WT and OPHN1 KO mice in the model of glial scar formation .

Taken together, these results show that OPHN1 does not have a major role in the structural development of astrocytes since the ramification of astroglial processes is unaltered in physiological conditions. However, astrocytes deficient for OPHN1 failed to display the typical structural plasticity normally observed after injury, which requires the recruitment of astrocytes and the elongation of their processes toward the wound proximal zone.

\section{Discussion}

We here found that the XLID protein OPHN1 inhibits the RhoA/ROCK/MLC2 pathway in astrocytes. We also showed that OPHN1 regulates the migration of astrocytes both in vitro and in vivo under injured conditions and contributes to glial scar formation in the adult.

\section{RhoA/ROCK/MLC2 pathway dysregulation in astrocytes in ID}

Small RhoGTPases are involved in neuronal network formation and synaptic plasticity that contribute to cognitive abilities (Ba et al., 2013; Benarroch, 2007; Newey et al., 2005; Ramakers, 2002; van Galen \& Ramakers, 2005). The dysregulation of those proteins is widely implicated in ID. It was indeed reported that in most pathological cases, RhoA is hyperactivated (Ramakers et al., 2012; Zamboni et al., 2018), although some cases of ID rather 
show a hypo-activation of the RhoA/ROCK/MLC2 pathway (Kutsche et al., 2000; Ravindran et al., 2017). These data indicate that this pathway, when unbalanced, leads to a pathological state. Here we found that the hyper-activation of this pathway in our OPHN1 KO mouse model of ID not only affects neurons, but also astrocytes. Remarkably, our results show that in our OPHN1 KO mouse of ID, hyper-activation of the RhoA/ROCK pathway contributes to an alteration of astrocyte migration in vitro and in vivo, associated with minor changes in their morphology. These data indicate that this pathway is essential for proper astrocytic behavior. RhoGTPases pathways are considered today as potential therapeutic targets to ameliorate and treat ID. In Rett syndrome, a severe encephalopathy affecting girls, modulation of RhoGTPases by the cytotoxic necrotizing factor 1 (CNF1) ameliorates the pathological state of astrocytes. This may contribute to the behavioral benefits of the treatment in the mouse model of the disease (De Filippis et al., 2012; De Filippis, Romano, \& Laviola, 2014). Here, we show that treatment of OPHN1 KO astrocytes with the Y27632 ROCK inhibitor not only restores the phosphorylation level of MLC2 to control conditions, but also rescues in vitro the migration deficit. As treatment with another ROCK inhibitor, Fasudil, improved some behavioral deficits in Ophn1 KO, it is tempting to speculate that part of the observed rescue is mediated by restoration of astrocytic function in the constitutive model (Meziane et al., 2016).

\section{OPHN1 functions in migrating astrocytes and in their morphology}

Regarding OPHN1 function in astrocytes migration, we found that OPHN1 binds to prolinerich proteins, which are involved in clathrin-mediated endocytosis (Khelfaoui et al., 2009). We further showed that constitutive transferrin receptor endocytosis is reduced in Ophn1 KO astrocytes, a deficit that is rescued by treatment with the Y27632 ROCK inhibitor. We also found that adhesion is not only increased in OPHN1 KO platelets (Fotinos et al., 2015), but also in astrocytes (unpublished data). We can hypothesize that loss-of-OPHN1 function alters the internalization of molecules such as integrins leading to an increase in cell adhesion and inhibition of astrocytic migration in stimulated conditions (Paul, Jacquemet, \& Caswell, 2015). More work however needs to be done to identify which integrins could mediate these effects. In addition to the migration deficit, we also observed that $\mathrm{KO}$ astrocytes at the migration front did not show a proper elongation and in vivo they have reduced branches complexity. It is difficult to know whether these morphological alterations were a cause or a consequence of the migration defect. F-actin cytoskeleton reorganization is required at the endocytic sites and alteration of RhoA/ROCK/MLC2 mediated contractility underneath the membrane may alter astroglial morphology. Since these changes were only observed upon injured conditions, OPHN1 may be required for astrocytic migration under challenging conditions such as scar formation.

\section{Altered glial scar formation in vivo in the cortex of OPHN1 KO mouse model of ID}

Scar formation is a common model used to study glial response, including changes in the morphology and recruitment of astrocytes at the wounded area. During glial scar formation, one of the cellular process that impact astrocytes is migration toward the lesion to isolate the wounded area from the healthy tissue (Sofroniew, 2009). As previously described, we observed two main regions around the lesion (Suzuki et al., 2012). The proximal region, where recruited astrocytes express nestin and are highly polarized, and the distal region where astrocytes express GFAP and have no specific polarized shape. With nestin immunohistochemistry, it is possible to observe elongated processes toward the lesion, as found in our model. It was recently shown that RhoA regulation by signal transducer and activator of transcription 3 (STAT3) altered glial scar formation in spinal cord injury (Renault-Mihara et al., 2017). Indeed, in the conditional KO mouse for STAT3, the upregulation of RhoA and the hyper-phosphorylation of its downstream effector MLC2 in vitro 
is associated with an alteration of astrocytic migration in vitro and with an astrocytic elongation defect toward the lesion in vivo. Remarkably, our model shows as well a hyperactivation of RhoA and a hyper-phosphorylation of its downstream effector MLC2. The alteration of the RhoA pathway also affected the establishment of the proximal region around the lesion, which is smaller, suggesting a migration and formation process defects in vivo mimicking the STAT3 KO model for this phenotype.

\section{Astrocyte versus neuronal dysfunction in OPHN1 linked ID}

Altogether, we used in vitro and in vivo challenging conditions to reveal some altered responses of OPHN1 KO astrocytes to these stress conditions. Could this astrocytic phenotype be secondary to the previously reported synaptic dysfunction after OPHN1 loss-offunction (Nadif Kasri, Nakano-Kobayashi, \& Van Aelst, 2011)? While this question could be addressed in vivo with the ophn 1 conditional inactivation in astrocytes, our in vitro model of astrocytic migration suggests that this astrocytic phenotype is cell-autonomous since there is no MAP2 positive neurons in our primary cell-culture model.

Is this astrocytic phenotype related to ID? We recently reviewed the astrocyte contribution to ID and highlight the importance of studying the tripartite synapse by dissecting each function in one cell type and also their interactions (Cresto et al., 2019). The observed altered response of Ophn1 KO astrocytes may participate to the neuronal defects. The interaction between astrocytic integrin at the membrane and the neuronal transmembrane Thy- $1 / \mathrm{CBP} / \mathrm{Csk} / \mathrm{Src}$ complex has been shown to trigger neurite retraction through the activation of RhoA/ROCK pathway in neurons (Maldonado et al., 2017). As OPHN1 may regulate the integrin internalization in astrocytes, dysregulation of the signal between the astrocyte and the neuron would notably alter the neuronal morphology in OPHN1 KO neurons especially the dendritic spines, whose density is reduced in the Ophn1 KO model (Khelfaoui et al., 2007). It is thus tempting to propose that alteration in astrocytic response to high stimulation could impact the neuronal structure and functions including synaptic activity and plasticity and, consequently, cognitive abilities. This nevertheless remains to be tested.

\section{References}

Abramoff, M. D., Megalhães, P. J., \& Ram, S. J. (2004). Image processing with ImageJ. Biophotonics International, 11(7), 36-42.

Allegra, M., Spalletti, C., Vignoli, B., Azzimondi, S., Busti, I., Billuart, P., . . . Caleo, M. (2017). Pharmacological rescue of adult hippocampal neurogenesis in a mouse model of X-linked intellectual disability. Neurobiol Dis, 100, 75-86. doi:10.1016/j.nbd.2017.01.003

Araujo, B. H. S., Kaid, C., De Souza, J. S., Gomes da Silva, S., Goulart, E., Caires, L. C. J., . . - Cavalheiro, E. A. (2018). Down Syndrome iPSC-Derived Astrocytes Impair Neuronal Synaptogenesis and the mTOR Pathway In Vitro. Mol Neurobiol, 55(7), 5962-5975. doi:10.1007/s12035-017-0818-6

Auer, M., Hausott, B., \& Klimaschewski, L. (2011). Rho GTPases as regulators of morphological neuroplasticity. Ann Anat, 193(4), 259-266. doi:10.1016/j.aanat.2011.02.015

Ba, W., van der Raadt, J., \& Nadif Kasri, N. (2013). Rho GTPase signaling at the synapse: implications for intellectual disability. Exp Cell Res, 319(15), 2368-2374. doi:10.1016/j.yexcr.2013.05.033 
Benarroch, E. E. (2007). Rho GTPases: role in dendrite and axonal growth, mental retardation, and axonal regeneration. Neurology, 68(16), 1315-1318. doi:10.1212/01.wnl.0000259588.97409.8f

Berger, A., Lorain, S., Josephine, C., Desrosiers, M., Peccate, C., Voit, T., . . . Bemelmans, A. P. (2015). Repair of rhodopsin mRNA by spliceosome-mediated RNA trans-splicing: a new approach for autosomal dominant retinitis pigmentosa. Mol Ther, 23(5), 918930. doi:10.1038/mt.2015.11

Billuart, P., Bienvenu, T., Ronce, N., des Portes, V., Vinet, M. C., Zemni, R., . . . Chelly, J. (1998). Oligophrenin-1 encodes a rhoGAP protein involved in X-linked mental retardation. Nature, 392(6679), 923-926. doi:10.1038/31940

Bosworth, A. P., \& Allen, N. J. (2017). The diverse actions of astrocytes during synaptic development. Curr Opin Neurobiol, 47, 38-43. doi:10.1016/j.conb.2017.08.017

Chen, F., \& LoTurco, J. (2012). A method for stable transgenesis of radial glia lineage in rat neocortex by piggyBac mediated transposition. J Neurosci Methods, 207(2), 172-180. doi:10.1016/j.jneumeth.2012.03.016

Chung, W. S., Welsh, C. A., Barres, B. A., \& Stevens, B. (2015). Do glia drive synaptic and cognitive impairment in disease? Nat Neurosci, 18(11), 1539-1545. doi:10.1038/nn.4142

Colin, A., Faideau, M., Dufour, N., Auregan, G., Hassig, R., Andrieu, T., . . . Deglon, N. (2009). Engineered lentiviral vector targeting astrocytes in vivo. Glia, 57(6), 667-679. doi:10.1002/glia.20795

Compagnucci, C., Barresi, S., Petrini, S., Billuart, P., Piccini, G., Chiurazzi, P., . . Z Zanni, G. (2016). Rho Kinase Inhibition Is Essential During In Vitro Neurogenesis and Promotes Phenotypic Rescue of Human Induced Pluripotent Stem Cell-Derived Neurons With Oligophrenin-1 Loss of Function. Stem Cells Transl Med, 5(7), 860869. doi:10.5966/sctm.2015-0303

Cresto, N., Pillet, L. E., Billuart, P., \& Rouach, N. (2019). Do Astrocytes Play a Role in Intellectual Disabilities? Trends Neurosci, 42(8), 518-527. doi:10.1016/j.tins.2019.05.011

Dallerac, G., \& Rouach, N. (2016). Astrocytes as new targets to improve cognitive functions. Prog Neurobiol, 144, 48-67. doi:10.1016/j.pneurobio.2016.01.003

De Filippis, B., Fabbri, A., Simone, D., Canese, R., Ricceri, L., Malchiodi-Albedi, F., . . . Fiorentini, C. (2012). Modulation of RhoGTPases improves the behavioral phenotype and reverses astrocytic deficits in a mouse model of Rett syndrome. Neuropsychopharmacology, 37(5), 1152-1163. doi:10.1038/npp.2011.301

De Filippis, B., Romano, E., \& Laviola, G. (2014). Aberrant Rho GTPases signaling and cognitive dysfunction: in vivo evidence for a compelling molecular relationship. Neurosci Biobehav Rev, 46 Pt 2, 285-301. doi:10.1016/j.neubiorev.2014.06.007

Delepine, C., Meziane, H., Nectoux, J., Opitz, M., Smith, A. B., Ballatore, C., . . Bienvenu, T. (2016). Altered microtubule dynamics and vesicular transport in mouse and human MeCP2-deficient astrocytes. Hum Mol Genet, 25(1), 146-157. doi:10.1093/hmg/ddv464

Dossi, E., Vasile, F., \& Rouach, N. (2018). Human astrocytes in the diseased brain. Brain Res Bull, 136, 139-156. doi:10.1016/j.brainresbull.2017.02.001

Etienne-Manneville, S. (2006). In vitro assay of primary astrocyte migration as a tool to study Rho GTPase function in cell polarization. Methods Enzymol, 406, 565-578. doi:10.1016/S0076-6879(06)06044-7

Etienne-Manneville, S., \& Hall, A. (2002). Rho GTPases in cell biology. Nature, 420(6916), 629-635. doi:10.1038/nature01148 
Fauchereau, F., Herbrand, U., Chafey, P., Eberth, A., Koulakoff, A., Vinet, M. C., . . . Billuart, P. (2003). The RhoGAP activity of OPHN1, a new F-actin-binding protein, is negatively controlled by its amino-terminal domain. Mol Cell Neurosci, 23(4), 574586. doi:10.1016/s1044-7431(03)00078-2

Fotinos, A., Klier, M., Gowert, N. S., Munzer, P., Klatt, C., Beck, S., . . Elvers, M. (2015). Loss of oligophrenin1 leads to uncontrolled Rho activation and increased thrombus formation in mice. J Thromb Haemost, 13(4), 619-630. doi:10.1111/jth.12834

Ghezali, G., Calvo, C. F., Pillet, L. E., Llense, F., Ezan, P., Pannasch, U., . . . Rouach, N. (2018). Connexin 30 controls astroglial polarization during postnatal brain development. Development, 145(4). doi:10.1242/dev.155275

Govek, E. E., Newey, S. E., Akerman, C. J., Cross, J. R., Van der Veken, L., \& Van Aelst, L. (2004). The X-linked mental retardation protein oligophrenin-1 is required for dendritic spine morphogenesis. Nat Neurosci, 7(4), 364-372. doi:10.1038/nn1210

Govek, E. E., Newey, S. E., \& Van Aelst, L. (2005). The role of the Rho GTPases in neuronal development. Genes Dev, 19(1), 1-49. doi:10.1101/gad.1256405

Hirrlinger, P. G., Scheller, A., Braun, C., Hirrlinger, J., \& Kirchhoff, F. (2006). Temporal control of gene recombination in astrocytes by transgenic expression of the tamoxifeninducible DNA recombinase variant CreERT2. Glia, 54(1), 11-20. doi:10.1002/glia.20342

Humeau, Y., Gambino, F., Chelly, J., \& Vitale, N. (2009). X-linked mental retardation: focus on synaptic function and plasticity. J Neurochem, 109(1), 1-14. doi:10.1111/j.14714159.2009.05881.x

Khelfaoui, M., Denis, C., van Galen, E., de Bock, F., Schmitt, A., Houbron, C., . . . Billuart, P. (2007). Loss of X-linked mental retardation gene oligophrenin1 in mice impairs spatial memory and leads to ventricular enlargement and dendritic spine immaturity. $J$ Neurosci, 27(35), 9439-9450. doi:10.1523/JNEUROSCI.2029-07.2007

Khelfaoui, M., Pavlowsky, A., Powell, A. D., Valnegri, P., Cheong, K. W., Blandin, Y., .. . Billuart, P. (2009). Inhibition of RhoA pathway rescues the endocytosis defects in Oligophrenin1 mouse model of mental retardation. Hum Mol Genet, 18(14), 25752583. doi:10.1093/hmg/ddp189

Kutsche, K., Yntema, H., Brandt, A., Jantke, I., Nothwang, H. G., Orth, U., . . . Gal, A. (2000). Mutations in ARHGEF6, encoding a guanine nucleotide exchange factor for Rho GTPases, in patients with X-linked mental retardation. Nat Genet, 26(2), 247250. doi:10.1038/80002

Lee, Y., Messing, A., Su, M., \& Brenner, M. (2008). GFAP promoter elements required for region-specific and astrocyte-specific expression. Glia, 56(5), 481-493. doi:10.1002/glia.20622

Loulier, K., Barry, R., Mahou, P., Le Franc, Y., Supatto, W., Matho, K. S., . . . Livet, J. (2014). Multiplex cell and lineage tracking with combinatorial labels. Neuron, 81(3), 505-520. doi:10.1016/j.neuron.2013.12.016

Maldonado, H., Calderon, C., Burgos-Bravo, F., Kobler, O., Zuschratter, W., Ramirez, O., . . . Leyton, L. (2017). Astrocyte-to-neuron communication through integrin-engaged Thy1/CBP/Csk/Src complex triggers neurite retraction via the RhoA/ROCK pathway. Biochim Biophys Acta Mol Cell Res, 1864(2), 243-254. doi:10.1016/j.bbamcr.2016.11.006

Matsumura, F., \& Hartshorne, D. J. (2008). Myosin phosphatase target subunit: Many roles in cell function. Biochem Biophys Res Commun, 369(1), 149-156. doi:10.1016/j.bbrc.2007.12.090

Meir, Y. J., Weirauch, M. T., Yang, H. S., Chung, P. C., Yu, R. K., \& Wu, S. C. (2011). Genome-wide target profiling of piggyBac and Tol2 in HEK 293: pros and cons for 
gene discovery and gene therapy. BMC Biotechnol, 11, 28. doi:10.1186/1472-6750$11-28$

Meziane, H., Khelfaoui, M., Morello, N., Hiba, B., Calcagno, E., Reibel-Foisset, S., . . . Billuart, P. (2016). Fasudil treatment in adult reverses behavioural changes and brain ventricular enlargement in Oligophrenin-1 mouse model of intellectual disability. Hum Mol Genet, 25(11), 2314-2323. doi:10.1093/hmg/ddw102

Milani, D., Ronzoni, L., \& Esposito, S. (2015). Genetic Advances in Intellectual Disability. $J$ Pediatr Genet, 4(3), 125-127. doi:10.1055/s-0035-1564438

Moortgat, S., Berland, S., Aukrust, I., Maystadt, I., Baker, L., Benoit, V., . . N Newbury-Ecob, R. A. (2018). HUWE1 variants cause dominant X-linked intellectual disability: a clinical study of 21 patients. Eur J Hum Genet, 26(1), 64-74. doi:10.1038/s41431-0170038-6

Nadif Kasri, N., Nakano-Kobayashi, A., Malinow, R., Li, B., \& Van Aelst, L. (2009). The Rho-linked mental retardation protein oligophrenin-1 controls synapse maturation and plasticity by stabilizing AMPA receptors. Genes Dev, 23(11), 1289-1302. doi:10.1101/gad.1783809

Nadif Kasri, N., Nakano-Kobayashi, A., \& Van Aelst, L. (2011). Rapid synthesis of the Xlinked mental retardation protein OPHN1 mediates mGluR-dependent LTD through interaction with the endocytic machinery. Neuron, 72(2), 300-315. doi:10.1016/j.neuron.2011.09.001

Nadif Kasri, N., \& Van Aelst, L. (2008). Rho-linked genes and neurological disorders. Pflugers Arch, 455(5), 787-797. doi:10.1007/s00424-007-0385-1

Nakano-Kobayashi, A., Tai, Y., Nadif Kasri, N., \& Van Aelst, L. (2014). The X-linked mental retardation protein OPHN1 interacts with Homer1b/c to control spine endocytic zone positioning and expression of synaptic potentiation. $J$ Neurosci, 34(26), 8665-8671. doi:10.1523/JNEUROSCI.0894-14.2014

Newey, S. E., Velamoor, V., Govek, E. E., \& Van Aelst, L. (2005). Rho GTPases, dendritic structure, and mental retardation. J Neurobiol, 64(1), 58-74. doi:10.1002/neu.20153

Paul, N. R., Jacquemet, G., \& Caswell, P. T. (2015). Endocytic Trafficking of Integrins in Cell Migration. Curr Biol, 25(22), R1092-1105. doi:10.1016/j.cub.2015.09.049

Pavlowsky, A., Chelly, J., \& Billuart, P. (2012). Major synaptic signaling pathways involved in intellectual disability. Mol Psychiatry, 17(7), 663. doi:10.1038/mp.2012.79

Petrelli, F., \& Bezzi, P. (2018). mGlu5-mediated signalling in developing astrocyte and the pathogenesis of autism spectrum disorders. Curr Opin Neurobiol, 48, 139-145. doi:10.1016/j.conb.2017.12.014

Raftopoulou, M., \& Hall, A. (2004). Cell migration: Rho GTPases lead the way. Dev Biol, 265(1), 23-32. doi:10.1016/j.ydbio.2003.06.003

Ramakers, G. J. (2002). Rho proteins, mental retardation and the cellular basis of cognition. Trends Neurosci, 25(4), 191-199. doi:10.1016/s0166-2236(00)02118-4

Ramakers, G. J., Wolfer, D., Rosenberger, G., Kuchenbecker, K., Kreienkamp, H. J., PrangeKiel, J., . . . Kutsche, K. (2012). Dysregulation of Rho GTPases in the alphaPix/Arhgef6 mouse model of X-linked intellectual disability is paralleled by impaired structural and synaptic plasticity and cognitive deficits. Hum Mol Genet, 21(2), 268-286. doi:10.1093/hmg/ddr457

Ravindran, E., Hu, H., Yuzwa, S. A., Hernandez-Miranda, L. R., Kraemer, N., Ninnemann, O., . . . Kaindl, A. M. (2017). Homozygous ARHGEF2 mutation causes intellectual disability and midbrain-hindbrain malformation. PLoS Genet, 13(4), e1006746. doi:10.1371/journal.pgen.1006746

Redolfi, N., Galla, L., Maset, A., Murru, L., Savoia, E., Zamparo, I., . . Lodovichi, C. (2016). Oligophrenin-1 regulates number, morphology and synaptic properties of adult-born 
inhibitory interneurons in the olfactory bulb. Hum Mol Genet, 25(23), 5198-5211. doi:10.1093/hmg/ddw340

Renault-Mihara, F., Mukaino, M., Shinozaki, M., Kumamaru, H., Kawase, S., Baudoux, M., . . Okano, H. (2017). Regulation of RhoA by STAT3 coordinates glial scar formation. J Cell Biol, 216(8), 2533-2550. doi:10.1083/jcb.201610102

Ridley, A. J. (2001). Rho GTPases and cell migration. J Cell Sci, 114(Pt 15), 2713-2722.

Ridley, A. J. (2015). Rho GTPase signalling in cell migration. Curr Opin Cell Biol, 36, 103112. doi:10.1016/j.ceb.2015.08.005

Ropers, H. H., \& Hamel, B. C. (2005). X-linked mental retardation. Nat Rev Genet, 6(1), 4657. doi:10.1038/nrg1501

Salvador-Carulla, L., Reed, G. M., Vaez-Azizi, L. M., Cooper, S. A., Martinez-Leal, R., Bertelli, M., . . . Saxena, S. (2011). Intellectual developmental disorders: towards a new name, definition and framework for "mental retardation/intellectual disability" in ICD-11. World Psychiatry, 10(3), 175-180. doi:10.1002/j.2051-5545.2011.tb00045.x

San Martin, A., \& Pagani, M. R. (2014). Understanding intellectual disability through

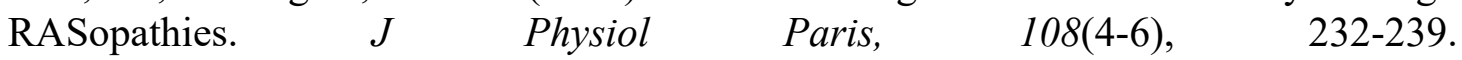
doi:10.1016/j.jphysparis.2014.05.003

Sofroniew, M. V. (2009). Molecular dissection of reactive astrogliosis and glial scar formation. Trends Neurosci, 32(12), 638-647. doi:10.1016/j.tins.2009.08.002

Stankiewicz, T. R., \& Linseman, D. A. (2014). Rho family GTPases: key players in neuronal development, neuronal survival, and neurodegeneration. Front Cell Neurosci, 8, 314. doi:10.3389/fncel.2014.00314

Suzuki, T., Sakata, H., Kato, C., Connor, J. A., \& Morita, M. (2012). Astrocyte activation and wound healing in intact-skull mouse after focal brain injury. Eur J Neurosci, 36(12), 3653-3664. doi:10.1111/j.1460-9568.2012.08280.x

Valnegri, P., Sala, C., \& Passafaro, M. (2012). Synaptic dysfunction and intellectual disability. Adv Exp Med Biol, 970, 433-449. doi:10.1007/978-3-7091-0932-8_19

van Galen, E. J., \& Ramakers, G. J. (2005). Rho proteins, mental retardation and the neurobiological basis of intelligence. Prog Brain Res, 147, 295-317. doi:10.1016/S0079-6123(04)47022-8

Vasile, F., Dossi, E., \& Rouach, N. (2017). Human astrocytes: structure and functions in the healthy brain. Brain Struct Funct, 222(5), 2017-2029. doi:10.1007/s00429-017-1383-5

Williams, E. C., Zhong, X., Mohamed, A., Li, R., Liu, Y., Dong, Q., . . Chang, Q. (2014). Mutant astrocytes differentiated from Rett syndrome patients-specific iPSCs have adverse effects on wild-type neurons. Hum Mol Genet, 23(11), 2968-2980. doi:10.1093/hmg/ddu008

Yusa, K., Zhou, L., Li, M. A., Bradley, A., \& Craig, N. L. (2011). A hyperactive piggyBac transposase for mammalian applications. Proc Natl Acad Sci U S A, 108(4), 15311536. doi:10.1073/pnas.1008322108

Zamboni, V., Jones, R., Umbach, A., Ammoni, A., Passafaro, M., Hirsch, E., \& Merlo, G. R. (2018). Rho GTPases in Intellectual Disability: From Genetics to Therapeutic Opportunities. Int J Mol Sci, 19(6). doi:10.3390/ijms19061821

Zegers, M. M., \& Friedl, P. (2014). Rho GTPases in collective cell migration. Small GTPases, 5, e28997. doi:10.4161/sgtp.28997

\section{Figure Legends}

Figure 1. OPHN1 regulates MLC phosphorylation during in vitro migration of astrocytes. a, Representative western blot illustrating OPHN1 expression in astrocyte cultures 
from WT and OPHN1 KO mice (mAstro), and in human control astrocytes differentiated from induced pluripotent stem cells (hAstro). b, Schematic drawing illustrating the role of OPHN1 in the RhoA/ROCK/MLC2 phosphorylation pathway. OPHN1 stimulates the GTPase activity of RhoA to its GDP bound inactivated form and turns-off the signaling from RhoA to ROCK and MLC2. Empty and red filled triangles, without or with phosphate residues. c, Western blot illustrating the phosphorylation of MLC in unscratched conditions ( $\varnothing$ ), and after 20 minutes and 24 hours following wounds on WT and OPHN1 KO astrocytes. d-e, Quantification of MLC2 phosphorylation levels in unscratched $(\varnothing)$ and scratched cells $(\mathrm{t}=20 \mathrm{~min}$ and $\mathrm{t}=24$ hours) (d: WT: $\mathrm{n}=5 ; \mathrm{KO}, \mathrm{n}=6, \mathrm{p}=0.0303$, Mann-Whitney-test and e: WT: $\mathrm{n}=5 ; \mathrm{KO}, \mathrm{n}=6, \mathrm{p}=0.0174$, two-way ANOVA, $\mathrm{t}=0 \mathrm{vs} \mathrm{t}=24 \mathrm{~h}$, post-hoc test $\mathrm{p}=0.0056$ ). $\mathbf{f}$, MLC2 phosphorylation after in vitro inactivation of the Ophnl gene. Astrocytes from Ophn1 conditional model (loxKO; Gfap-CreERT2) were treated with 4OH-T for 1 or 4 days to induce Ophnl gene inactivation and ROCK activation was studied by monitoring the phosphorylation of MLC2. g, Quantification of OPHN1 expression (in green) and MLC2 phosphorylation levels (in blue) in untreated $(\mathrm{t}=0)$ and $4 \mathrm{OH}-\mathrm{T}$ treated cells ( $\mathrm{t}=1$ and $\mathrm{t}=4$ days) (OPHN1 reduction: WT: $\mathrm{n}=3 ; \mathrm{KO}, \mathrm{n}=3, \mathrm{p}=0.0264$, ANOVA-test, untreated vs 4 days, MLC2 phosphorylation: WT: $\mathrm{n}=3 ; \mathrm{KO}, \mathrm{n}=3, \mathrm{p}=0.0001$, ANOVA-test, untreated vs 4 days). Asterisks indicate statistical significance $\left({ }^{*} p<0.05,{ }^{* *} p<0.01,{ }^{* * *} p<0.001\right)$.

Figure 2. Migration of OPHN1 deficient astrocytes is altered and is rescued by treatment with a ROCK inhibitor. a, Image J software Spectrum pictures of $0 \mathrm{~h}$ and $24 \mathrm{~h}$ wounded WT and OPHN1 KO astrocytes and schematic illustrations of the measurements. Scale bar: $100 \mu \mathrm{m}$. b, Quantification of migration of WT and OPHN1 KO astrocytes $24 \mathrm{~h}$ after the wound in untreated $(-)$ or Y27632-treated $(0.1 \mu \mathrm{M})(+)$ conditions (WT: $\mathrm{n}=9 ; \mathrm{KO}: \mathrm{n}=11$ for both conditions, ANOVA-test, $\mathrm{p}=0.0041$, Bonferroni's multiple comparisons test). c, MLC2 phosphorylation in untreated and Y27632 treated $\mathrm{KO}$ astrocytes ( $\mathrm{n}=5$ for each condition). d, Quantification of MLC2 phosphorylation in untreated (-) and treated (+) conditions both in WT and KO astrocytes. (WT: $n=5$ and KO: $n=5$ for each condition, ANOVA-test, $p=0.0136$, Bonferroni's multiple comparisons test). Data were normalized to untreated WT condition. Asterisks indicate statistical significance $\left(* p<0.05,{ }^{* *} p<0.01\right)$.

Figure 3. The morphology of migrating astrocytes is altered in OPHN1 deficient cells. a, Representative pictures illustrating vinculin immunostaining of migrating WT and OPHN1 $\mathrm{KO}$ astrocytes at the migration front of the scar $24 \mathrm{~h}$ after scratching. Scale bar: $100 \mu \mathrm{m}$. Examples of major and minor axis measured with the ImageJ software are represented in yellow. b, Frequency distribution of area, major and minor axis lengths of WT $(n=320)$ and OPHN1 KO $(\mathrm{n}=402)$ migrating astrocytes c, Means of area, major and minor axis lengths of migrating WT $(n=320)$ and OPHN1 KO $(n=402)$ astrocytes (Area, $\mathrm{p}=0.0027$, t-test with Welch's correction; $\mathrm{p}=0.0032$, KS test, major: $\mathrm{p}=0.0002$, t-test with Welch's correction; $\mathrm{p}=0.012$, KS test, minor: $\mathrm{p}=0.4358$, t-test with Welch's correction; $\mathrm{p}=0.7009, \mathrm{KS}$ test). Asterisks indicate statistical significance $(* *: p<0.01, * * *: p<0.001)$.

Figure 4. Unchanged morphology of OPHN1 astrocytes in vivo in the cortex of adult mice under physiological conditions. a, Vector scheme (see Materials and Methods section for description). b, GFP labeled astrocytes in the cortex at postnatal days 21 after in utero electroporation of embryos at day 15.5. The electroporated cells express Glutamine Synthase (GS) and S100ß markers at postnatal day 21. c.c.: corpus callosum. Scale bars, $500 \mu \mathrm{m}$ (upper panel), $10 \mu \mathrm{m}$ (lower panel). c, Sholl analysis in astrocytes from WT $(\mathrm{n}=23$ cells from 3 embryos) and OPHN1 KO ( $\mathrm{n}=29$ cells from 4 embryos) mice ( $\mathrm{p}=0.5846$ for two-way ANOVA). 
Figure 5. Altered morphology of OPHN1 astrocytes in vivo in the cortex of adult mice after wound. a, Schematics illustrating the glial scar formation model in the cortex of 2month-old mice induced by a needle in the cortex, also used to inject AAVs expressing GFP in astrocytes. b, Representative pictures illustrating the specificity of the GFP labeling in astrocytes using the GFAP marker (red). Scale bars, $150 \mu \mathrm{m}$ (upper panel), $10 \mu \mathrm{m}$ (lower panel). c, Representative picture illustrating the Sholl analysis based on GFP cytoplasmic staining of astrocytes in the distal zone of the wound. Scale bar, $10 \mu \mathrm{m}$. d, Quantification of the Sholl analysis in astrocytes from WT $(n=30)$ and OPHN1 KO $(n=30)$ mice $(p<0.0001$ for two-way ANOVA-test and Bonferroni's post-hoc). Asterisks indicate statistical significance $(*: p<0.05)$.

Figure 6. Glial scar formation is altered in adult OPHN1 deficient mice. a, Schematics and representative pictures illustrating the glial scar model and the nestin immunostaining in the cortex. b, Top, representative pictures illustrating nestin immunostaining of the cortical wounded area in the proximal zone of the scar in WT and OPHN1 KO mice. Calibration bar: purple $=0$, white $=255$. Bottom, quantification of normalized proximal zone area in OPHN1 WT $(n=5)$ and KO $(n=5)$ mice $(p=0.0335$, t-test $)$. c, Top: representative pictures of DAPI staining near the cortical wound in WT mice. Bottom: quantification of DAPI positive nucleus density near the cortical lesion in WT $(n=5)$ and OPHN1 KO $(n=4)$ mice $(p=0.2892$, t-test). d, Top: representative pictures of Caspase 3 and e, phosphohistone 3 immunostainings in the wounded area for identification of cells in apoptosis and proliferative state, respectively. Bottom, corresponding quantification of cell density per $\mathrm{mm}^{2}$ in $\mathrm{WT}(\mathrm{n}=5)$ and OPHN1 KO $(n=4)$ wounded areas (caspase $3: p=0.6250$, t-test; phospho-histone3: $p=0.5090$, $\mathrm{t}$-test). Asterisks indicate statistical significance $(*: p<0.05)$. Scale bars: $200 \mu \mathrm{m}$.

Figure S1. OPHN1 KO astrocytes have normal MLC2 levels in physiological conditions. a, Representative western blot of MLC2 expression in unscratched condition normalized to GAPDH expression. b, Quantification of MLC2 level in WT $(n=6)$ and OPHN1 KO astrocytes $(n=6)$. No statistical difference was found between the two genotypes $(p=0.98$, Mann-Whitney-test).

Figure S2. Dose-response curve of the ROCK inhibitor Y27632 on MLC2 phosphorylation. a, Representative western blot of MLC2 phosphorylation and expression in cultured wild type astrocytes treated with various concentrations of Y27632 (0.1, 1 and 10 $\mu \mathrm{M})$. GAPDH was used as a loading control. b, Quantification of MLC2 phosphorylation in these different conditions (Y27632: $\mathrm{n}=3$ for $0,0.1,1$ and $10 \mu \mathrm{M}, \mathrm{p}=0.0007$, one-way ANOVA test and Bonferroni's multiple comparison). Asterisks indicate statistical significance (*: $p<0.05, * *: p<0.01)$. Among the three tested concentrations $(0.1,1$ and $10 \mu \mathrm{M})$, only the smallest one had no effect on MLC2 phosphorylation and was chosen for rescue experiments of OPHN1 KO astrocytes in the in vitro migrating assay.

Figure S3. OPHN1 KO astrocytes at the rear of the scar do not show altered cell area. a, Representative pictures of vinculin immunostaining in WT and OPHN1 KO astrocytes in vitro. Scale bar: $100 \mu \mathrm{m}$. b, Quantification of astrocytic area in WT $(\mathrm{n}=80)$ and OPHN1 KO $(n=80)$ conditions $(p>0.99$, t-test $)$. 

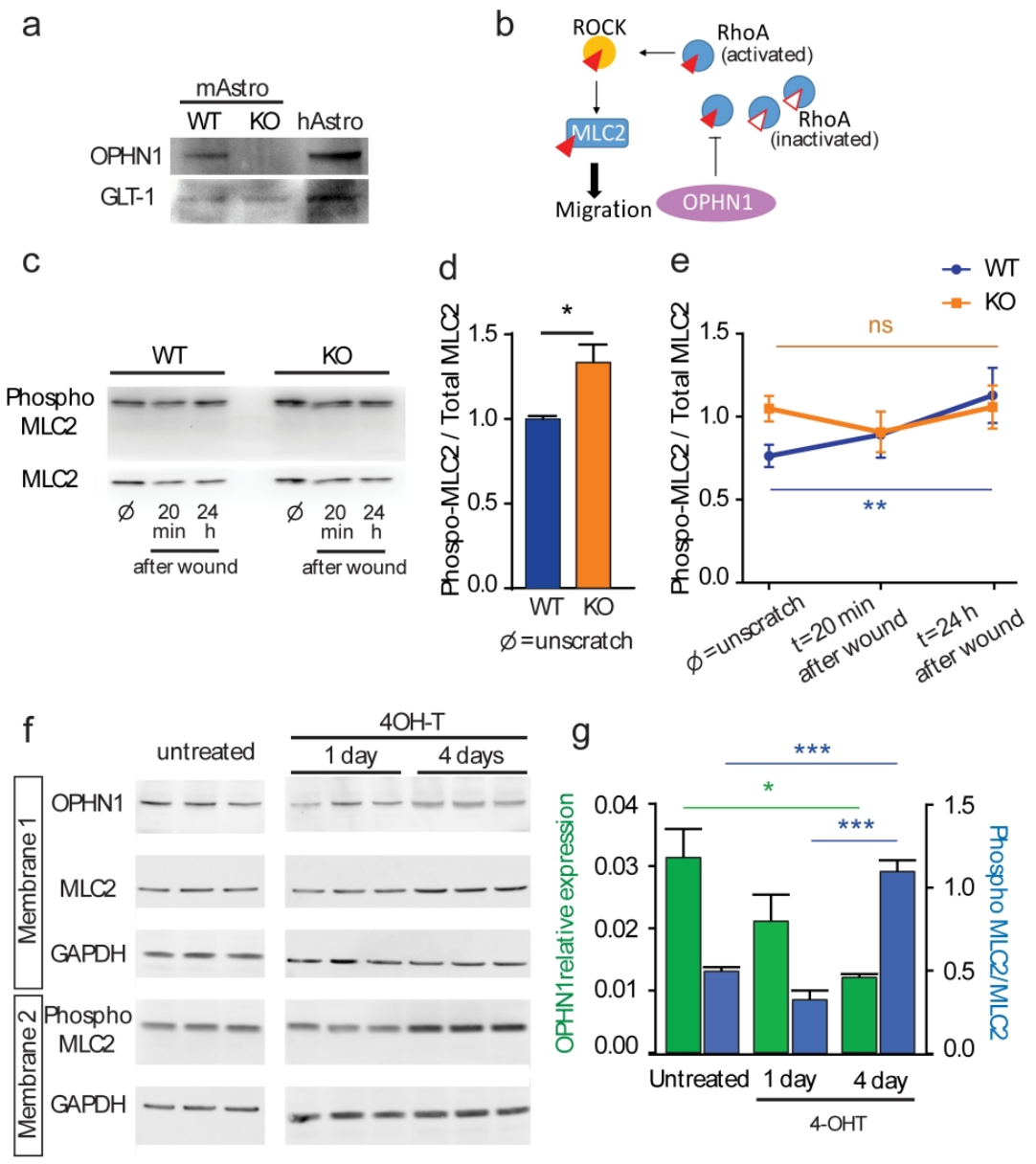

Figure 1: OPHN1 regulates MLC phosphorylation during in vitro migration of astrocytes. 

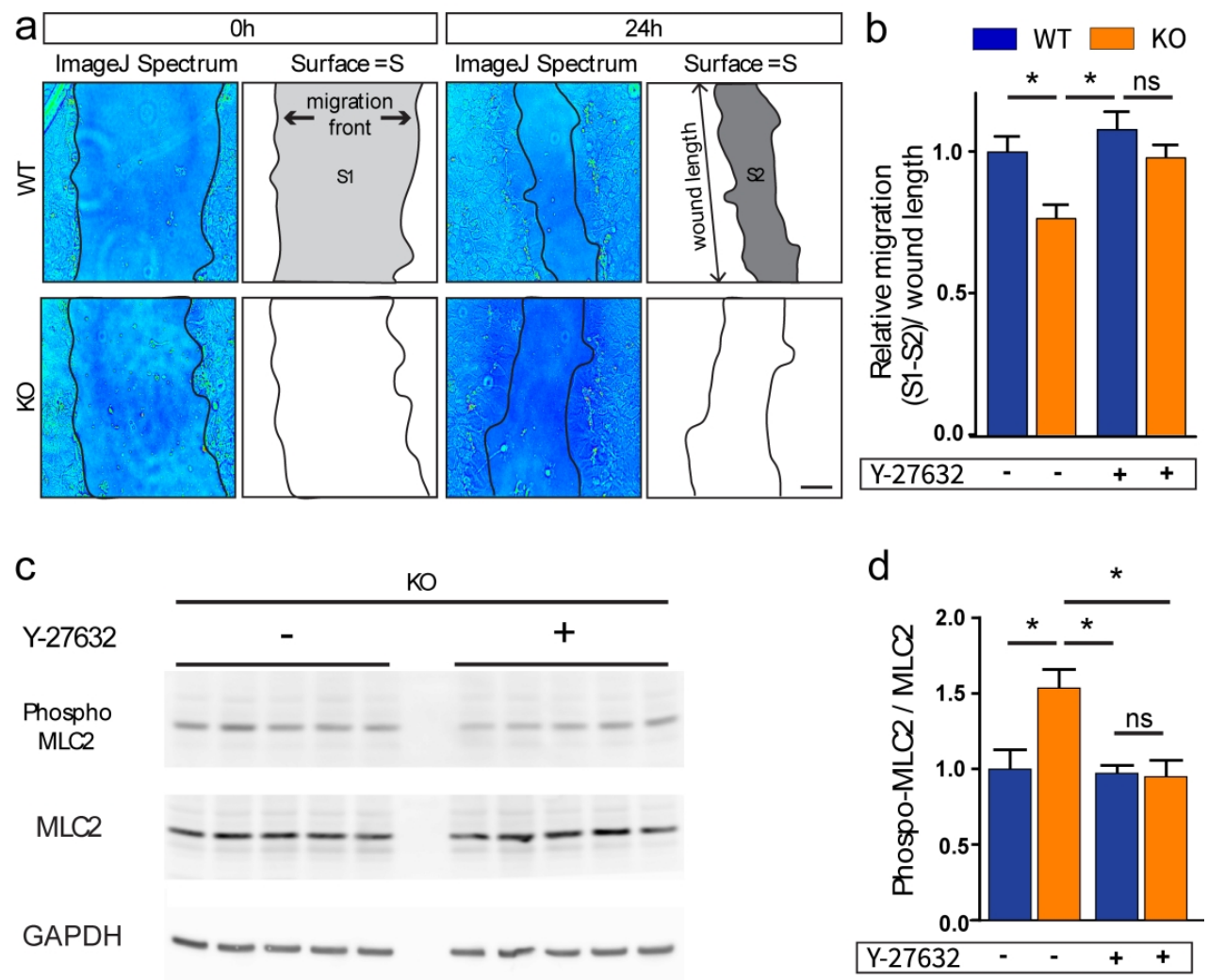

Figure 2. Migration of OPHN1 deficient astrocytes is altered and is rescued by treatment with a ROCK inhibitor. 

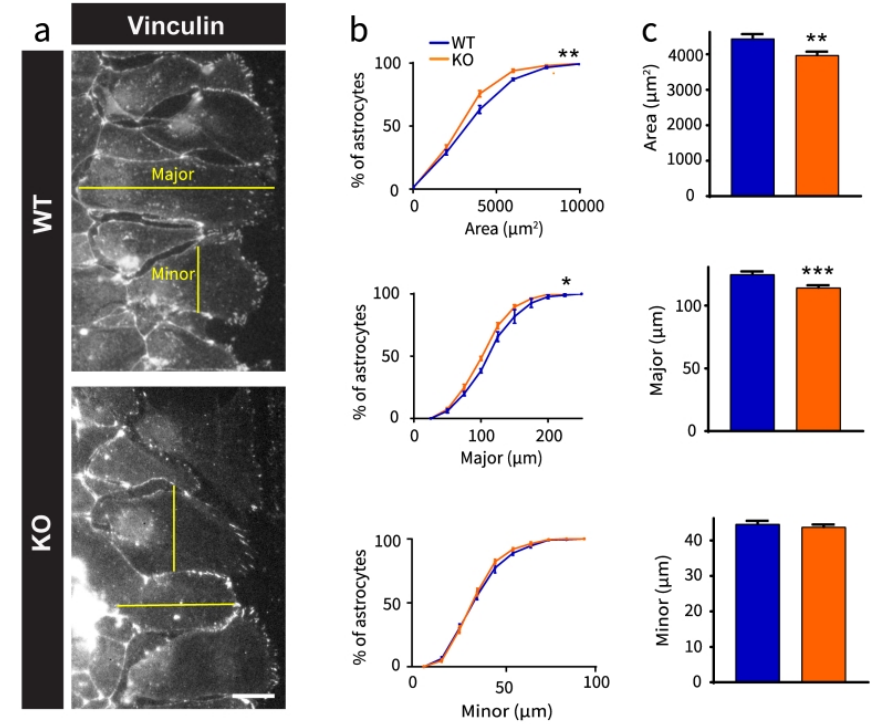

Figure 3: The morphology of migrating astrocytes is altered in OPHN1 deficient cells. 


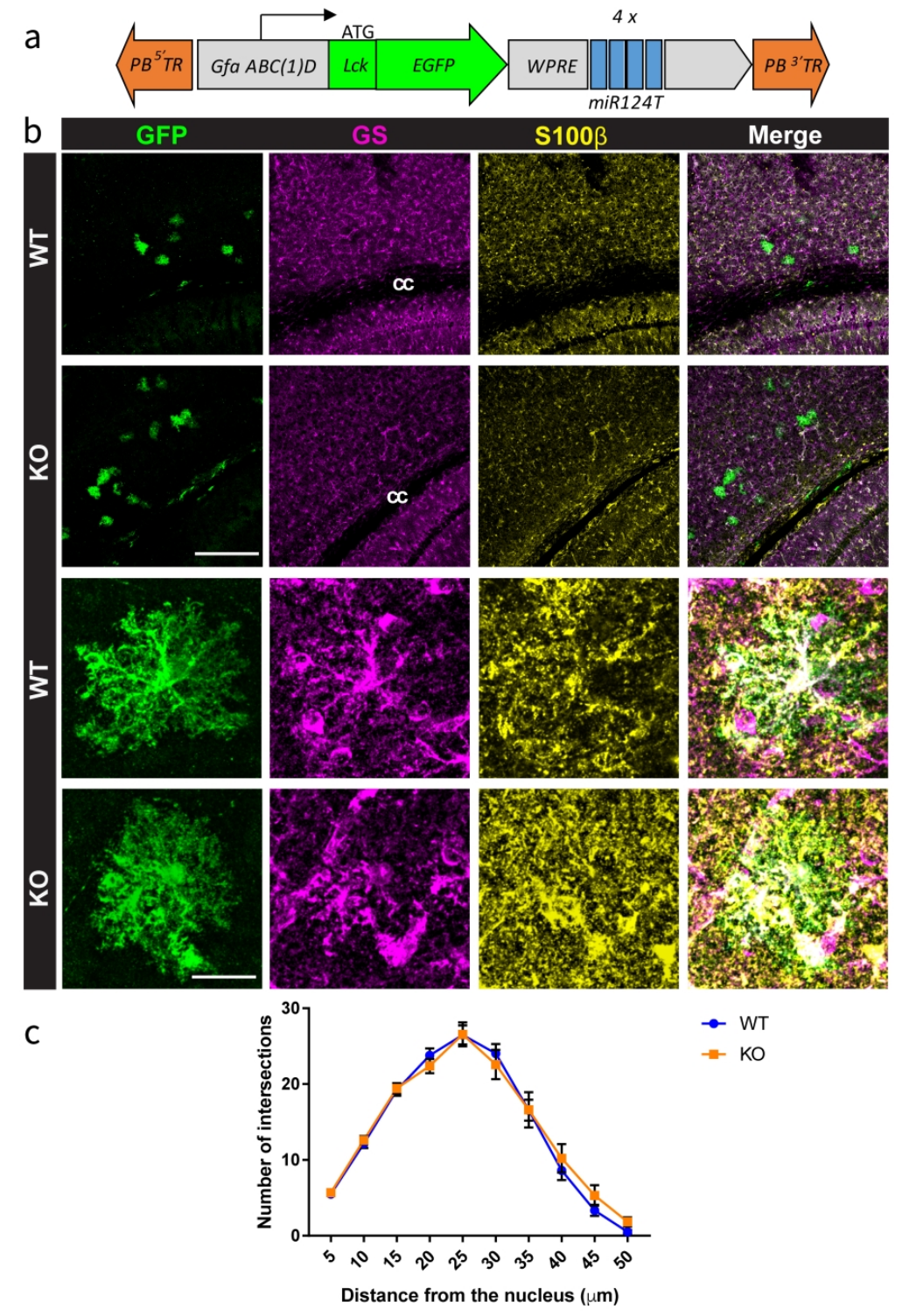

Figure 4: Unchanged morphology of OPHN1 astrocytes in vivo in the cortex of adult mice under physiological conditions. 


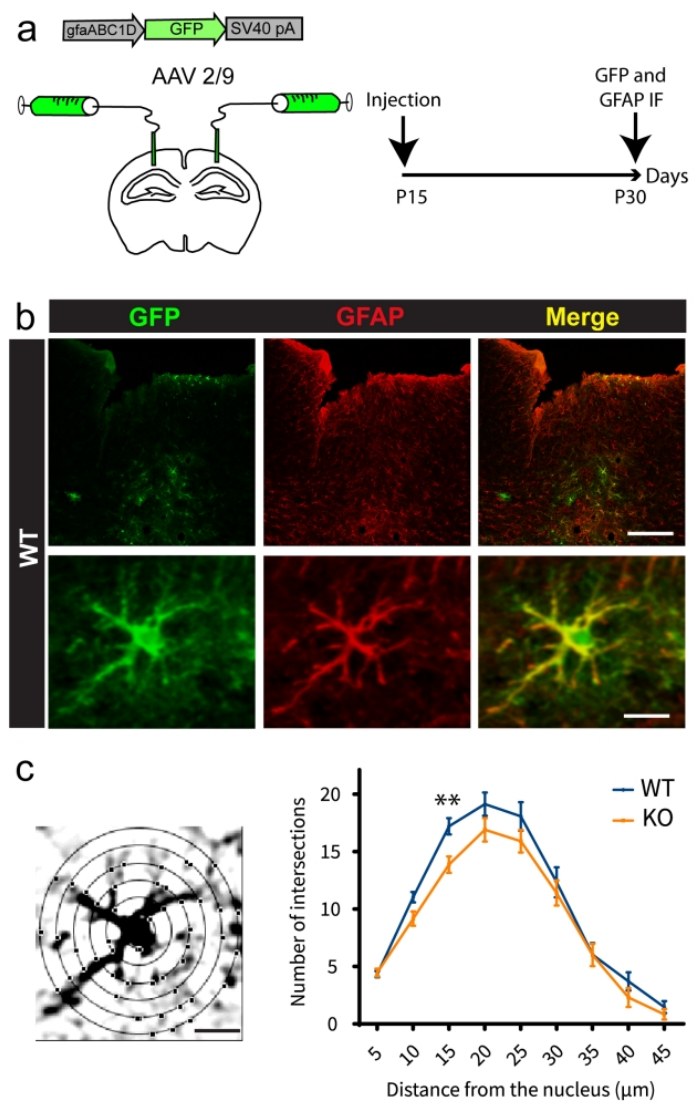

Figure 5. Altered morphology of OPHN1 astrocytes in vivo in the cortex of adult mice after wound. 

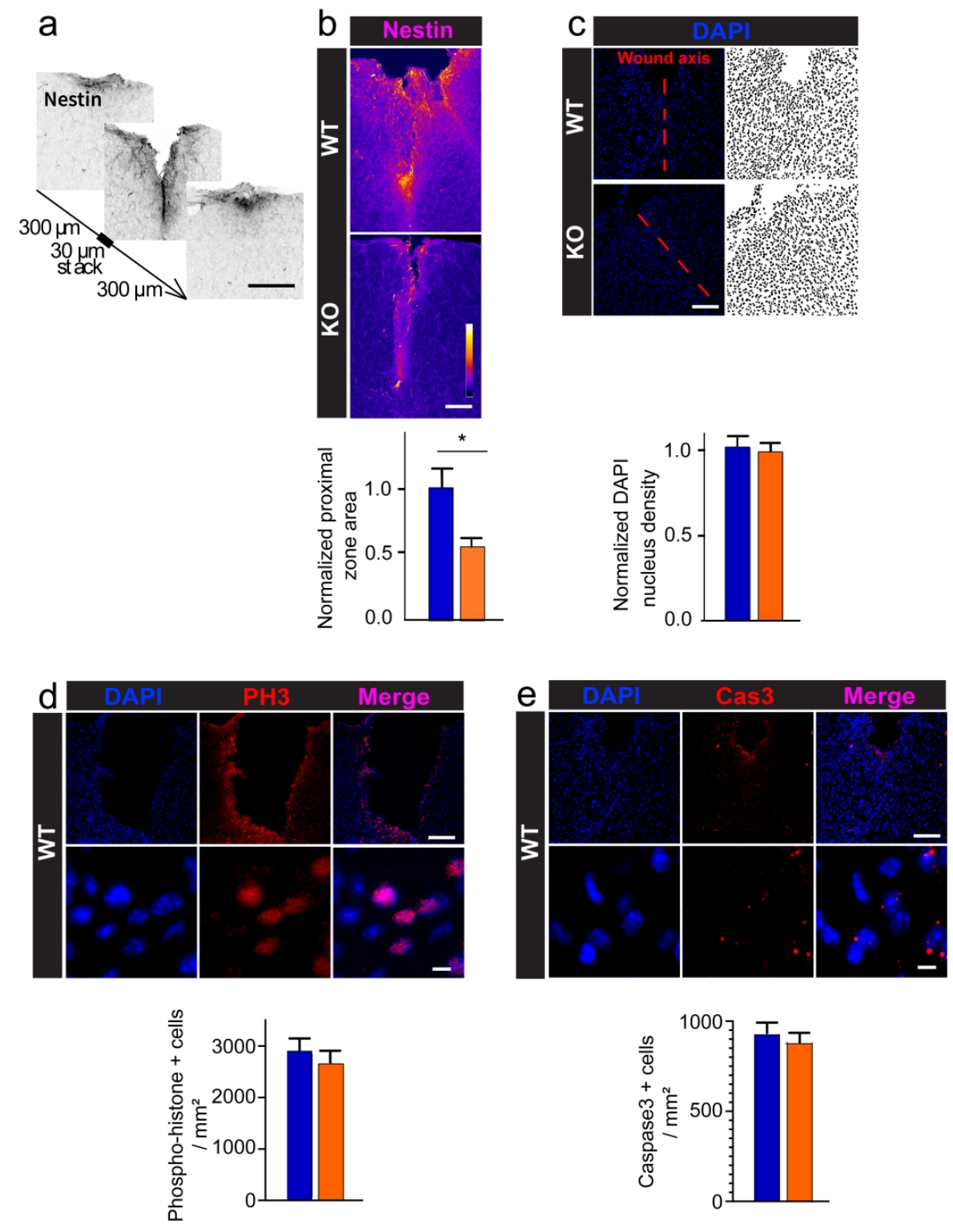

Figure 6: Glial scar formation is altered in adult OPHN1 deficient mice. 

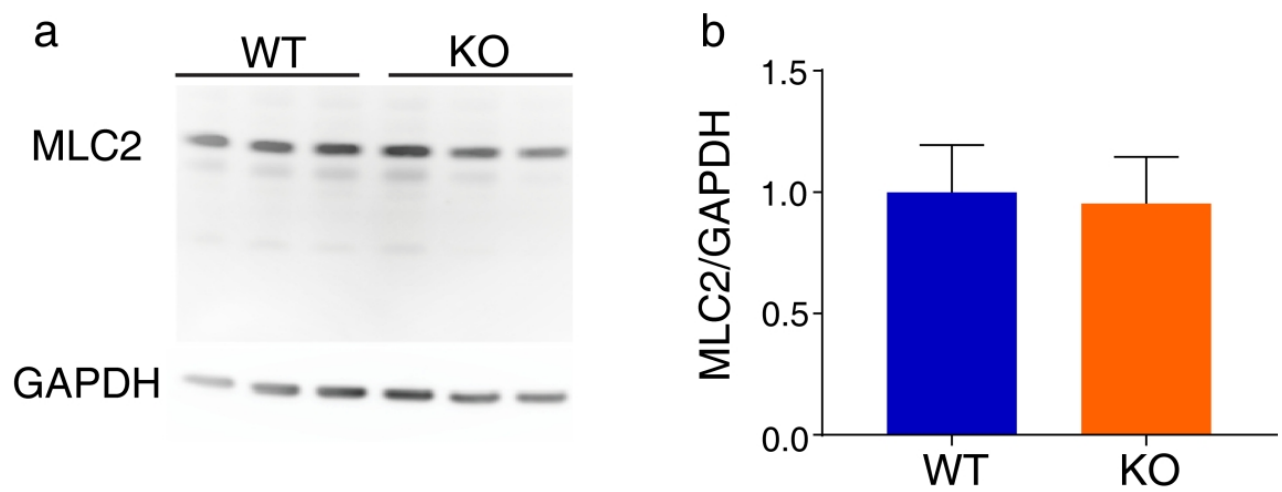

Figure S1. OPHN1 KO astrocytes have normal MLC2 levels in physiological conditions. 


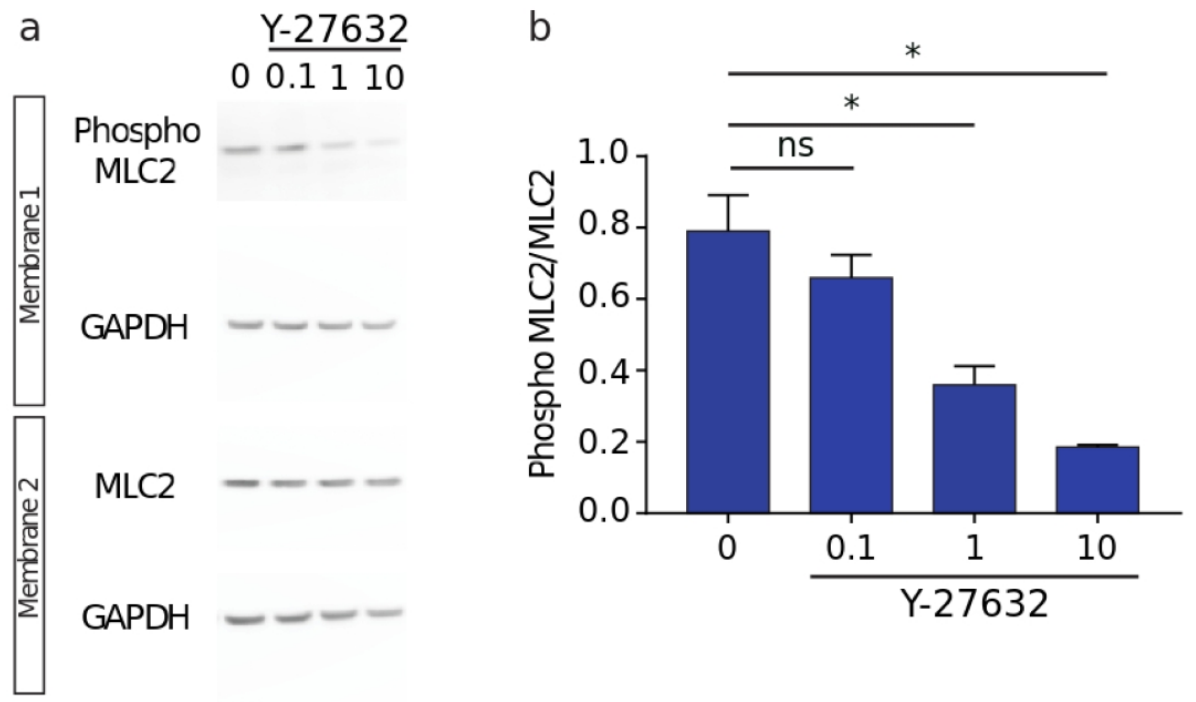

Figure S2: Dose-response curve of the ROCK inhibitor Y27632 on MLC2 phosphorylation. 

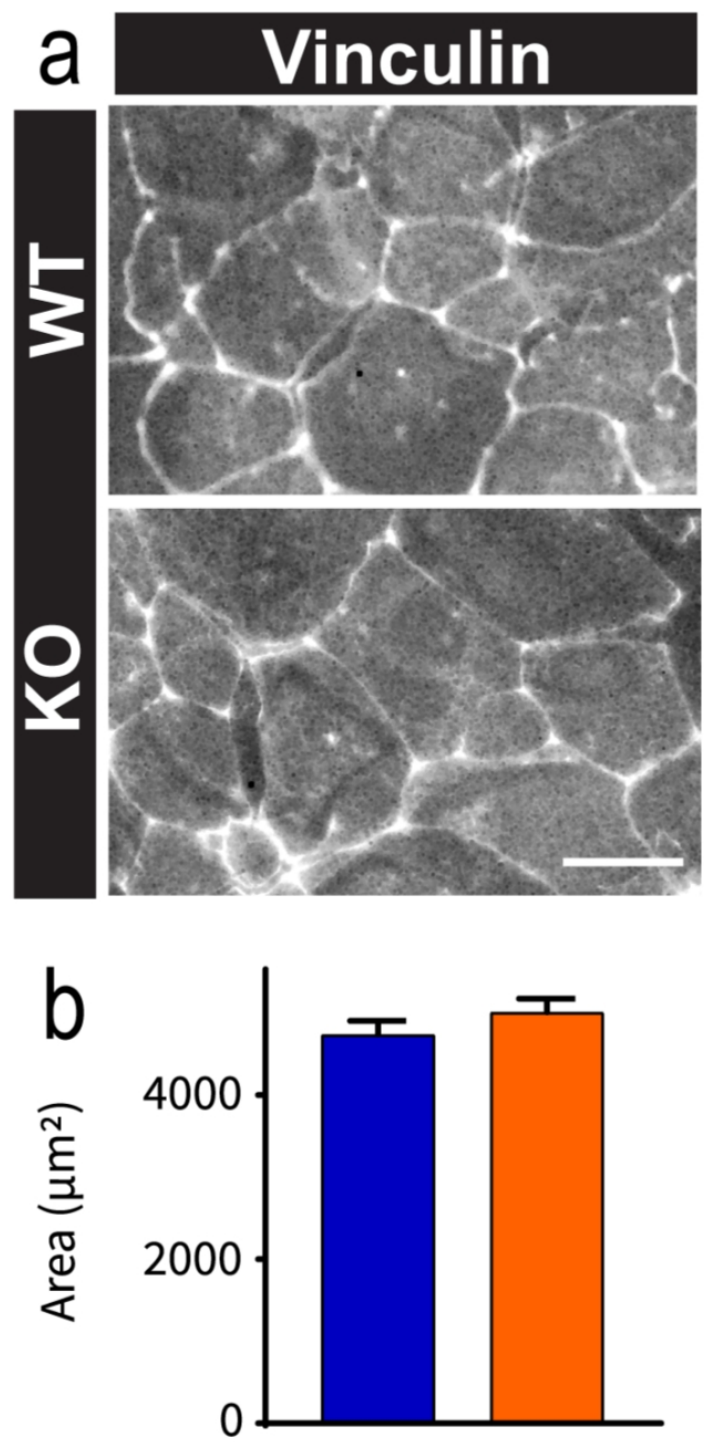

Figure S3: OPHN1 KO astrocytes at the rear of the scar do not show altered cell area. 